\title{
Childhood N-Methyl-D-Aspartic Acid Receptor (NMDAR) Antibody Mediated Encephalitis
}

\section{Lim}

Bibliography

DOI http://dx.doi.org/

$10.1055 / s-0031-1295420$

Neuropediatrics 2011;

42: $177-178$

(c) Georg Thieme Verlag KG

Stuttgart · New York

ISSN 0174-304X

Correspondence

Dr. M. Lim, BMBS, PhD

Consultant Paediatric Neurologist

Evelina Children's Hospital

@ Guys and St Thomas' NHS

Foundation Trust

Kings Health Partners Academic

Health Science Centre

Lambeth Palace Road

London SE1 7EH

Ming.lim@gstt.nhs.uk
Antibody mediated encephalitides, where patients present with neurological syndromes associated with antibodies directed against ion channels, receptors and other synaptic proteins are now well recognised in adults and children [8]. Autoantibodies reported in children predominantly target the voltage-gated potassium channel (VGKC)-complex proteins and N-Methyl-D-Aspartic acid receptor (NMDAR).

The identification of antibodies directed against the NMDA receptor in young women with ovarian teratoma presenting with a syndrome of memory deficits, psychiatric symptoms, decreased level of consciousness and hypoventilation lead to the recognition of a novel paraneoplastic syndrome [9]. Termed anti-NMDAR encephalitis by the original authors, patients commonly present with an initial cortical stage with a range of seizures, psychiatric features, and cognitive dysfunction; progressing often after 10-20 days to a subcortical stage when a movement disorder, autonomic dysfunction and depressed level of consciousness begin to manifest $[3,6]$.

Importantly, children form a substantial subgroup of these patients $[4,5]$. Whilst they appear to share similar clinical features to adult patients, children have a significantly lower risk of an underlying malignancy. In a comprehensive single national reference centre study of patients presenting with anti-NMDAR encephalitis, the risk of a tumour ranged from $56 \%$ in women $>18$ years old to $9 \%$ in girls 14 years old and under [4]. This study also demonstrated that paraclinical features appear to mirror that of adult patients. The cerebrospinal fluid (CSF) is frequently abnormal in children consisting of a lymphocytosis (87\%), hyperproteinaemia (30\%) and presence of oligoclonal bands (83\%). Brian MRI is abnormal in up to $30 \%$ of patients and abnormalities often representing signal intensity either on FLAIR (Fluid attenuated inversion recovery) or T2 images in one or more areas (medial temporal lobe, periventricular, cerebellar). The electroencephalogram (EEG) demonstrates epileptic activity in $30 \%$ of patients, and focal or diffuse slowing is seen in majority of the other patients.

As with most novel entities, reports of a broadening phenotype follow. Children with a dyskinetic from of encephalitis lethargica were demonstrated to have NMDAR antibodies [2]. Other neurological syndromes ranging from psychosis, pure epilepsy syndromes, limbic encephalitis and other encephalitides have been reported to be associated with NMDAR antibodies [reviewed in ref [6]]. More recently NMDAR antibodies have also been reported in a child with late onset regression autism [1].

In this issue, Goldberg et al., report a previouslyhealthy 9-year-old girl presenting with focal non-convulsive status epilepticus (NCSE) accompanied by focal signs (right hemianopsia) and neuroimaging with cerebrospinal fluid positivity for the NMDAR antibody. This is the first report of NMDAR encephalitis presenting as focal NCSE. Importantly, as the authors point out, clinicians should consider an autoimmune aetiology even in a focal neurological presentation. Indeed, similar focality and/or laterality have been reported in an NMDAR antibody mediated movement disorder [7], and other autoimmune neurological disorders (like lupus, thyroid antibody associated encephalopathy).

As we start to recognise NMDAR encephalitis and its variants, clinicians are now tasked at how best to manage these patients. Early immunosuppression and its benefits are now established in its management $[3,6]$. In the acute phase, corticosteroids with additional immunoglobulins (IVIG) or, if justified by clinical severity, plasma exchange are recommended by Dalmau and colleagues as first line therapy. In patients with a delayed response, treatment is escalated to Rituximab (as in Goldberg et al. case) or cyclophosphamide (or both) [3]. Responses to immunotherapy in both, children and adults, can be slow (over months) and variable, often requiring prolonged immunosuppression, a multidisciplinary team input including physical rehabilitation and psychiatric management of protracted behavioural symptoms. Although a large proportion of early onset patients were reported to have had a full or substantial recovery at 1 year after immunotherapy or tumour removal [4], longer follow-up is required to fully evaluate the neuro-cognitive sequelae of this condition. Furthermore, $30 \%$ of adult [5] and $25 \%$ of childhood and adolescent onset patients [4] with NMDAR-associated encephalitis relapse. A more effective management strategy may require immunosuppression to be extended beyond symptom remission, with the appropriate use of steroidsparing immunosuppressive agents like azathioprine and methotrexate [6]. Despite the lower risk of malignancy in children, a protracted tumour surveillance period appears prudent, particularly if the treatment response is poor or relapses occur, as is recommended in adult patients [3].

As such, refining the treatment regime to optimise outcome and prevent relapse remains a huge challenge. Current therapeutic strategies 
require systematic evaluation in children with NMDAR and other autoantibody mediated conditions. Additional neuroprotective strategies and therapies to attenuate disease specific pathogenic mechanisms may be required to treat these patients more effectively.

\section{References}

1 Creten C, van der Zwaan S, Blankespoor RJ et al. Late onset autism and anti-NMDA-receptor encephalitis. Lancet 2011; 378 (9785): 98

2 Dale RC, Irani SR, Brilot F et al. N-methyl-D-aspartate receptor antibodies in pediatric dyskinetic encephalitis lethargica. Ann Neurol 2009; 66 (5): 704-709

3 Dalmau J, Lancaster E, Martinez-Hernandez E et al. Clinical experience and laboratory investigations in patients with anti-NMDAR encephalitis. Lancet Neurol 2011; 10 (1): 63-74
4 Florance NR, Davis RL, Lam C et al. Anti-N-methyl-D-aspartate receptor (NMDAR) encephalitis in children and adolescents. Ann Neurol 2009; 66 (1): 11-18

5 Irani SR, Bera $K$, Waters $P$ et al. N-methyl-D-aspartate antibody encephalitis: temporal progression of clinical and paraclinical observations in a predominantly non-paraneoplastic disorder of both sexes. Brain 2010; 133 (Pt 6): 1655-1667

6 Irani SR, Vincent A. NMDA receptor antibody encephalitis. Curr Neurol Neurosci Rep 2011; 11 (3): 298-304

7 Rubio-Agustí I, Dalmau J, Sevilla T et al. Isolated hemidystonia associated with NMDA receptor antibodies. Mov Disord 2011; 26 (2): 351-352

8 Vincent A, Bien CG, Irani SR et al. Autoantibodies associated with diseases of the CNS: new developments and future challenges. Lancet Neurol 2011; 10 (8): 759-772

9 Vitaliani R, Mason W, Ances B et al. Paraneoplastic encephalitis, psychiatric symptoms, and hypoventilation in ovarian teratoma. Ann Neurol 2005; 58 (4): 594-604 